\title{
Exploiting White Spaces for Karachi through Artificial Intelligence: Comparison of NARX and Cascade Feed Forward Back Propagation
}

\author{
Shabbar Naqvi ${ }^{1}$, Minaal Ali ${ }^{2}$, Aamir Zeb Shaikh ${ }^{3}$, Yamna Iqbal ${ }^{4}$, Abdul Rahim ${ }^{5}$, Saima Khadim ${ }^{6}$, Talat Altaf ${ }^{7}$ \\ Department of Computer Systems Engineering, Balochistan University of Engineering and Technology Khuzdar, Pakistan ${ }^{1}$ \\ Department of Electronic Engineering, NED University of Engineering and Technology Karachi \\ Pakistan 2, 3, 4,5 \\ Department of Telecommunications Engg, Dawood University of Engineering and Technology, Karachi. Pakistan ${ }^{6}$ \\ Department of Electrical Engineering, Sir Syed University of Engineering and Technology Karachi, Pakistan ${ }^{7}$
}

\begin{abstract}
Marriage of Internet of Everything (IoE) and Cognitive Radio driven technologies seems near under the umbrella of $6 \mathrm{G}$ and $6 \mathrm{G}+$ communication standard. The expected new services that will be introduced in $6 \mathrm{G}$ communication will require high data rates for transmission. The learning based algorithms will play a key role towards successful implementation of these novel technologies and evolving next generation wireless standards for providing ubiquitous connectivity. This paper investigates performance of two artificial neural network (ANN) based algorithms for Karachi. These include Nonlinear autoregressive exogenous Algorithm (NARX) and cascade feed forward back propagation neural network (CFFBNN) scheme. A dataset for Karachi is also developed for $1805 \mathrm{MHZ}$. The results of the two algorithms are compared that show Mean Square Error (MSE) for CFFBNN is 6.8877e-5 at epoch 16 and MSE for NARX is 3.1506e-11 at epoch 26. Hence, exploiting computational performance, NARX performs much superior than the classis CFFBNN algorithm.
\end{abstract}

Keywords-6G; cognitive radio; NARX; cascaded feed forward neural network; learning

\section{INTRODUCTION}

Internet of Things (IoT) typically refers to interconnection of various networks. The basic network may include some or all of the sensing entities such as kitchens, personal computers etc. into sensing entities [1], [2]. Internet of Everything (IoE) refers to the concept of people to machine connection, people to data and data to things. Hence, it is expected to require huge amount of bandwidth as the data requirement is towards higher side. After the roll out of $5 \mathrm{G}$ communication standard, researchers are exploring and investigating different tools and technologies so that the requirements could be fulfilled for next generation wireless standard i.e. 6G. Hence, many applications are envisioned such as wireless brain computer interactions, autonomous systems and connected robotics, block chain and distributed ledger technologies, multisensory RF applications [3], big data for 6G, AI enabled closed loop and intelligent wireless communications [4]. These services are expected to be provided through technologies such as above $6 \mathrm{G}$ for $6 \mathrm{G}$, transceivers with integrated frequency bands, Edge Artificial Intelligence, Integrated terrestrial airborne and satellite networks, energy transfer and harvesting and beyond 6G technologies [3]. Additionally, a paradigm shift from software to network intelligence is also expected into the next generation of wireless networks [4]. The evolution towards intelligent connections will result in data rates of up to $1 \mathrm{~Tb} / \mathrm{s}$, highly energy efficient with a battery-free IoT device option, massively low latency control i.e. less than $1 \mathrm{msec}$ i.e. end- to end latency, broad frequency bands $73 \mathrm{GHz}-140 \mathrm{GHz}$ and $1-3$ $\mathrm{THz}$, ubiquitous connectivity through integration of global cellular and satellite systems and connected intelligence with machine learning capability[4].

Artificial intelligence algorithms can be distributed between two set of schemes i.e. machine learning and deep learning techniques[5], [6]. These algorithms are computationally efficient algorithms that observe the action rather than computing complex equations. So, based on the observations these algorithms predict the future results. As the wireless communication scenarios are mostly random in nature, hence, these algorithms will help in making the system autonomous. And the algorithms are selected with least MSE. Hence, a list of algorithms are available to automate various levels of wireless communications[5]. In this paper two algorithms are investigated for Karachi RF spectrum measurements. These are NARX and CFFBNN. The results show that the NARX algorithm performs better than CFFBNN.

The rest of the paper is as under. Section II discusses the NARX and CFFBNN, Section III presents the related work, Section IV shows the simulation Results and discussion Section V presents the conclusion of the paper.

\section{NARX AND CFFBNN}

Literature shows that the ANNs have proved to be very efficient for time series and modeling data in various fields including financial, communication network traffic prediction, chaotic time prediction and also for noisy data.

\section{A. Nonlinear Autoregressive Exogenous Scheme}

NARX, a type of nonlinear model proposed by Leontaritis and billings is a nonlinear model. It is used for estimating the future values of the time series based on its outputs and exogenous input.

A feature of nonlinear auto regressive models with exogenous inputs (NARX) recurrent neural network is that 
their architecture has limited feedback, which is dependent only on output neurons and not on hidden neurons. NARX networks are computationally powerful. That is why NARX models are considered to predict a wide class of nonlinear behaviors.

\section{NARX is a discrete-time nonlinear system [7].}

The typical architecture of NARX dynamic neural network contains three layers namely input, hidden and output. Unlike other types of dynamic networks like Elaman and Layer recurrent network, there is no context layer. Also that the output is feedback to input in this type of network [8]. In terms of configurations used for NARX network, Fig. 1 Shows two commonly used configurations as found in the literature. In series parallel architecture, an open loop mechanism is deployed which means that only true inputs are used to predict the future values of time series data and estimated output values are not fed back as inputs for this purpose. In completely parallel architecture, output values are fed back as input for prediction of output of time series data. In general, series parallel architecture is used during the training phase of the system and Parallel architecture is used for multistep ahead prediction [9].

\section{B. Cascade Feed forward Backpropagation Neural Network}

ANN functions like human neuron which is interconnected to one another. A generic neural network consists of many layers namely input layers, hidden layers and output layers. CFFNN is one of the design types used to develop neural networks. In CFFNN, neurons are connected with both preceding layers as well as all the neurons in layers. The theory behind the vast number of connections is that more connections provide better learning capability for the proposed ANN setup. These networks also used back propagation algorithm for updating weights. In this aspect they are similar to feed forward network. Different algorithms are used to change the weights of the networks. Common examples are Bayesian and Marquardt algorithms. Cascade feed forward network is a type of network which is considered to be efficient as well as flexible. Fast learning is also a property associated with Cascade feed forward network [10].

Another example of Cascade feed forward network is shown in Fig. 4. It can be seen that input neuron is connected to hidden neuron and hidden neuron has connection not only with input neuron but also with output neuron [12].

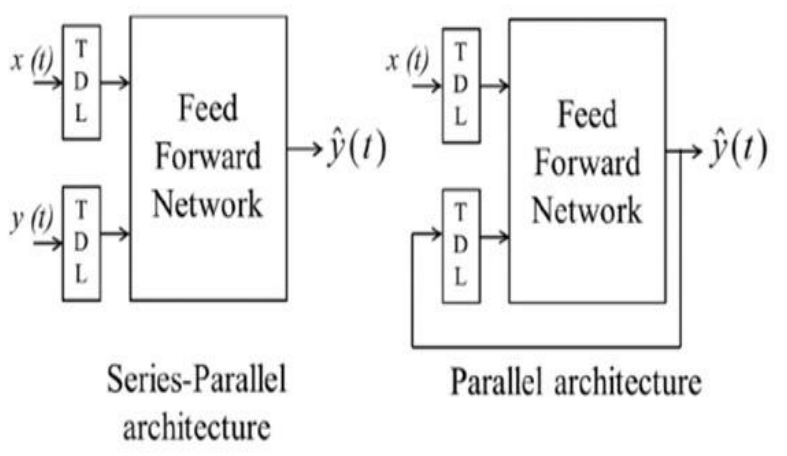

Fig. 1. Series Parallel Architecture of NARX Model [11].

\section{RELATED WORK}

Cascade feed forward networks have also been used in Cognitive Radio technology.

Iliya et al. have used Cascade feed forward network in a series of experiments along with other algorithms for the prediction of real world RF power within the GSM 900, Very High Frequency (VHF) and Ultra High Frequency (UHF) FM and TV bands. Back propagation algorithm was used in Cascade feed forward network. Authors used sensitivity analysis in order to reduce the input vectors of the prediction models. Experiments showed that Cascade Feed forward network outperformed in terms of Average Mean Square Error (AMSE) for 30 independent runs and their standard deviation [13].

In [14], authors explore the secondary use of RF spectrum under LTE cellular environment. Power spectral density (PSD) of randomly chosen primary user signals is calculated. The prediction performance of the proposed setup is compared using NARX and Auto-regressive integrated moving average (ARIMA). Mean Square Error (MSE) is taken as performance criteria. Additionally, authors also proposed hybrid system comprising of sequential combination of ARIMA followed by NARX. Authors compare the performance of proposed hybrid scheme with ARIMA and NARX. The results of the simulation setup show that hybrid model performs better than NARX by $15.15 \%$ and $9.68 \%$ than ARIMA in some cases while $40 \%$ better than NARX and $33.33 \%$ than ARIMA in other cases. The proposed study recommends that the behavioral modeling of licensed activity under LTE setup can prove to be highly useful for secondary users. Especially, due to the fact that no a prior information regarding primary users is required. Hence, it is recommended that the proposed setup can be used by opportunistic users to exploit licensed bands in secondary fashion for optimal use of RF spectrum.

IP based connections typically show a dynamic traffic requirement behavior. This is due to the fact that different applications such as live video conferencing, audio communication, image transmission etc. require different amount of bandwidth. Some of these applications require higher bandwidth requirement than others such as Quality of Service (QoS) based transmissions and video communications. Hence, to resolve the imminent issue of bandwidth requirement, authors in [15] proposed smart VPN bonding scheme. In this scheme, more channels are bonded together to fulfill the requirements of end user. Additionally, the proposed scheme also provides better load balancing. Throughput of the proposed setup is measured that shows promising results.

Cognitive Users initiate the transmission in secondary fashion by following cognitive cycle. That includes spectrum sensing, decision making to shift the transmission, interference testing and etc. In [11], authors propose a sensing-transmission scheduling scheme. The performance of various artificial neural network based architectures is compared under periodic data as well as non-repeating data. The algorithms considered are NARX, feed forward, focused time delay, Elman, distributed time delay, cascade feed forward back propagation and layer recurrent network. The comparison between cascade feed forward back propagation neural network (CFBPNN) and 
NARX neural network is presented in the paper. For periodically repeating data, NARX algorithm takes 17.32 seconds in comparison to CFBPNN that takes 95.79 seconds to complete the operation. The optimal percentage achieved through NARX is $97.86 \%$ and CFBPNN 95.79\%. For nonrepeating data, NARX achieves $98.16 \%$ using 7.65 seconds in comparison to achieving $100 \%$ from 3.83 seconds by CFBPNN. Additionally, MSE for CFBPNN is achieved at 0.0091743 in comparison to 0.088502 by NARX.

Cognitive Radio is a novel concept that promises to solve the imminent issue of spectrum scarcity through opportunistic use. Cognitive Radio may operate on many different schemes. These include underlay, overlay and interweave radio schemes [16].

In underlay cognitive radio environment, primary users and unlicensed cognitive users both exist at the same time. However, the coexistence can only be achieved successfully if the users are following the set rules. Two of such issues are addressed by the authors in [17]. These include the interference limit from primary network for the secondary user network and the amount of interference; secondary users are creating for primary users. The issue of interference can be addressed through a learning algorithm such that the sensor should continually detect the amount of RF power and report to central station. And the central station should have the rights to allow or prevent the unused spectral spaces based on the interference limit.

In [18] authors use NARX based classifier to detect the tumors. The feature extraction is done through Principal Component Analysis (PCA). Additionally, back propagation is used to train the proposed network and NARX is used to make classifications. The results of the proposed setup suggest that the NARX has great potential to detect tumors.

In [19], authors use NARX to predict various time series cases. Additionally, a comparison between real and artificial chaotic data from various experiments is presented. Three conclusions are drawn from the proposed simulation of network. The NARX has a potential to capture the nonlinear dynamic behavior of proposed setup. Another concluding remark is that these algorithms also have some limitations such as challenges in learning long time dependencies due to vanishing gradient. Additionally, these algorithms have limitation of optimizing the embedded memory. Furthermore, Recurrent Neural Network (RNN) model highly affects the performance of prediction. Hence, it is suggested that the user should always avoid the over fitting and saturation condition because too many hidden layers lead to poor prediction.

In this paper, NARX and CFFBNN are compared for Karachi dataset. The algorithms will help in improving the opportunistic activity for Karachi city based on the permission from the concerned departments in the country. For this purpose, a dataset is also developed by using NI 2901 USRP devices. The secondary activity is already allowed by FCC in USA in broadcast bands. The next section explains the details of the experimentation and analysis of the simulation results.

\section{Simulation Results}

This section presents the results of the two algorithms selected and simulated for Karachi RF spectrum measurements. The frequency of operation is selected as 1805 MHz. Fig. 2 shows the performance metric for cascade feed forward back propagation neural network. This includes training, validation, test and best results for the algorithm. The MSE for the proposed setup comes out to be is $6.8877 \mathrm{e}-5$ at epoch 16. Fig. 3 shows the performance of learning based algorithm i.e. NARX for the proposed setup. The MSE results for the proposed setup come out to be 3.1506e-11 at epoch 26 . Thus, showing a $61 \%$ increase in the epochs for achieving an excellent MSE results. The results clearly suggest that the performance of NARX algorithm supersedes its opposite algorithm; however, this performance is achieved on the basis of more training time. Epoch represents the number of passes the algorithm sees the data set. One epoch is equal to one forward and one backward pass. Hence, it can be concluded that MSE performance and no. of epochs are showing a trade-off.

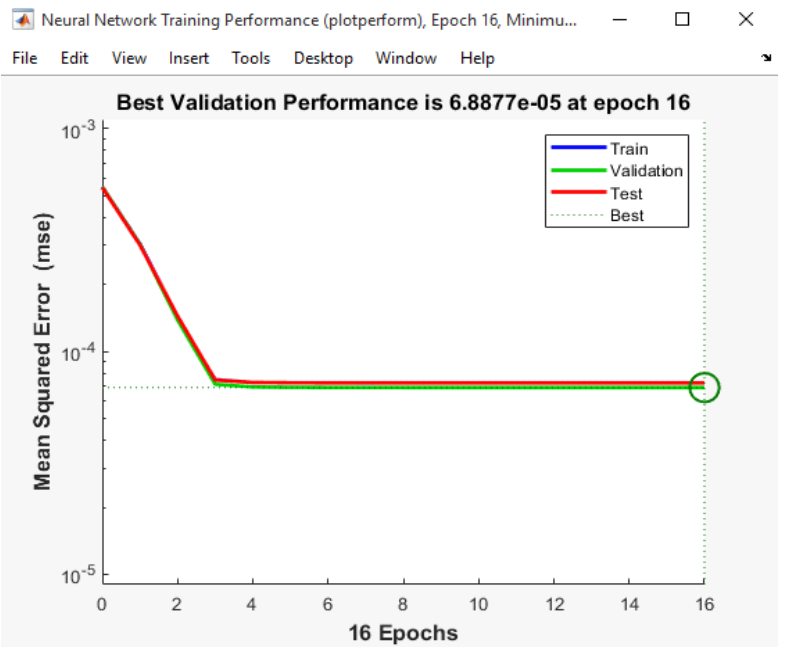

Fig. 2. Shows the Performance Results of Cascade Feed forward back Propagation Neural Network.

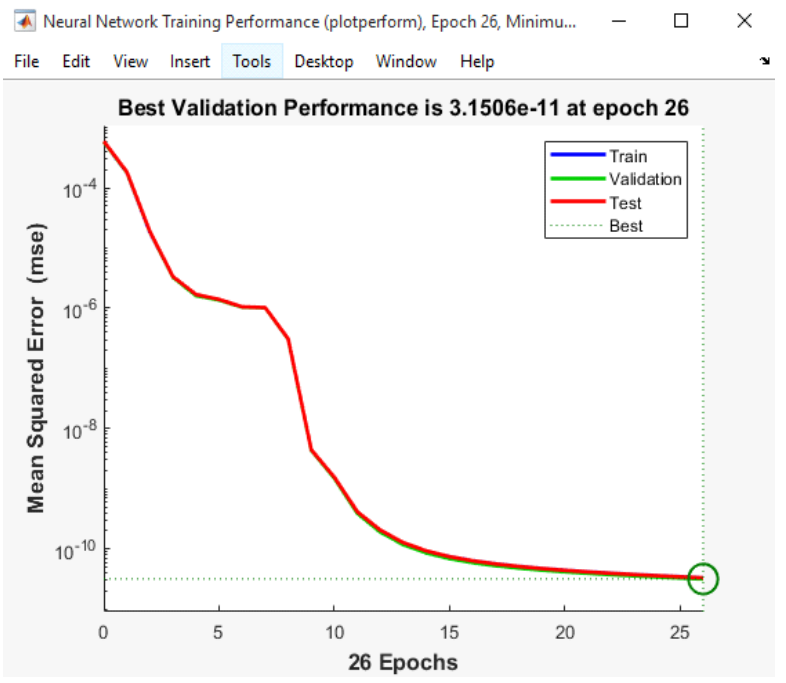

Fig. 3. Shows the Performance Results of NARX. 


\section{CONCLUSION}

Performance analysis of two machine learning algorithms i.e. NARX and CFFBPNN is analyzed and compared for Karachi city. The results of the proposed network will allow the future secondary users of the wireless network to exploit the RF spectrum in opportunistic fashion such that a ubiquitous connectivity could be provided even under the usage of existing RF network. The proposed algorithm will be highly useful especially for $6 \mathrm{G}$ and $6 \mathrm{G}+$ wireless communication standards. NARX algorithm takes 26 epochs for producing the best possible statistics regarding training and validation while CFFBNN produces the best statistics n 16 epochs. However the CFFBNN results in MSE of 6.8877e-5 in comparison to MSE produced by NARX that comes out to be $3.15 \mathrm{e}-11$. Hence, the prediction results advocate the use of NARX algorithm in future generation wireless radios with autonomous capabilities in comparison to CFFBNN.

The future work will be to investigate Deep Learning algorithms for prediction of spectral holes in Karachi. Additionally, the task will be to compare the performance between NARX, CFFBNN and Deep Learning based algorithms. The focus will be to recommend best possible algorithms, so that secondary use of RF spectrum can be successfully implemented in Karachi.

\section{ACKNOWLEDGMENT}

The authors would like to thank the Administration of NED University of Engineering \& Technology, Karachi, Pakistan, for providing resources to complete this research.

\section{REFERENCES}

[1] M. H. Miraz, M. Ali, P. S. Excell, and R. Picking, "A review on Internet of Things (IoT), Internet of everything (IoE) and Internet of nano things (IoNT)," in 2015 Internet Technologies and Applications (ITA), 2015, pp. 219-224.

[2] A. A. Khan, A. Z. Shaikh, S. Naqvi, and T. Altaf, "A Novel Cognitive Radio Enabled IoT System for Smart Irrigation," J. Inform. Math. Sci., vol. 9, no. 1, pp. 129-136, 2017.

[3] W. Saad, M. Bennis, and M. Chen, "A vision of $6 \mathrm{G}$ wireless systems: Applications, trends, technologies, and open research problems," IEEE Netw., 2019.

[4] K. B. Letaief, W. Chen, Y. Shi, J. Zhang, and Y.-J. A. Zhang, "The roadmap to 6G: AI empowered wireless networks," IEEE Commun. Mag., vol. 57, no. 8, pp. 84-90, 2019.
[5] C. Jiang, H. Zhang, Y. Ren, Z. Han, K.-C. Chen, and L. Hanzo, "Machine learning paradigms for next-generation wireless networks," IEEE Wirel. Commun., vol. 24, no. 2, pp. 98-105, 2016.

[6] M. A. Khan, A. Z. Shaikh, S. Naqvi, S. Khadim, and T. Altaf, "Deep Learning Enabled Spectrum Sensing Radio for Opportunistic Usage," IJCSNS, vol. 19, no. 11, p. 179, 2019.

[7] H. Xie, H. Tang, and Y.-H. Liao, "Time series prediction based on NARX neural networks: An advanced approach," in 2009 International conference on machine learning and cybernetics, 2009, vol. 3, pp. 12751279.

[8] M. Saberivahidaval and S. Hajjam, "Comparison between performances of different neural networks for wind speed forecasting in $\mathrm{P}$ ayam airport, I ran," Environ. Prog. Sustain. Energy, vol. 34, no. 4, pp. 1191-1196, 2015.

[9] J.-A. Ryu and S. Chang, "Data Driven Heating Energy Load Forecast Modeling Enhanced by Nonlinear Autoregressive Exogenous Neural Networks," 2019.

[10] I. Khan, H. Zhu, D. Khan, and M. K. Panjwani, "Photovoltaic Power prediction by Cascade forward artificial neural network," in 2017 International Conference on Information and Communication Technologies (ICICT), 2017, pp. 145-149.

[11] I. K. Aulakh, "ANN Application in Sensing-Transmission Scheduling in Cognitive Radio."

[12] G. Renisha and T. Jayasree, "Cascaded Feedforward Neural Networks for speaker identification using Perceptual Wavelet based Cepstral Coefficients," J. Intell. Fuzzy Syst., no. Preprint, pp. 1-13, 2019.

[13] S. Iliya, E. Goodyer, J. Gow, J. Shell, and M. Gongora, "Application of artificial neural network and support vector regression in cognitive radio networks for RF power prediction using compact differential evolution algorithm," in 2015 federated conference on computer science and information systems (FedCSIS), 2015, pp. 55-66.

[14] R. T. Fleifel, S. S. Soliman, W. Hamouda, and A. Badawi, "LTE primary user modeling using a hybrid ARIMA/NARX neural network model in CR," in 2017 IEEE Wireless Communications and Networking Conference (WCNC), 2017, pp. 1-6.

[15] G. Capizzi et al., "Available bandwidth estimation in smart VPN bonding technique based on a NARX neural network," in 2017 Federated Conference on Computer Science and Information Systems (FedCSIS), 2017, pp. 601-606.

[16] A. Z. Shaikh and L. Tamil, "Cognitive radio enabled telemedicine system," Wirel. Pers. Commun., vol. 83, no. 1, pp. 765-778, 2015.

[17] F. S. Mohammadi and A. Kwasinski, "Neural network cognitive engine for autonomous and distributed underlay dynamic spectrum access," ArXiv Prepr. ArXiv180611038, 2018.

[18] H. P. H. Anh, "Medical Image Classification and Symptoms Detection Using Fuzzy NARX Technique," in 4th International Conference on Biomedical Engineering in Vietnam, 2013, pp. 335-342.

[19] E. Diaconescu, "The use of NARX neural networks to predict chaotic time series," Wseas Trans. Comput. Res., vol. 3, no. 3, pp. 182-191, 2008. 